\title{
La innovación de las empresas turísticas en EI Salvador
}

\author{
Rocío Estéfani Portillo de Reyes ${ }^{1}$ \\ Víctor Hugo Quintana García ${ }^{2}$ \\ Ada Roxana Torres de Acevedo ${ }^{3}$ \\ Docentes investigadores, Facultad de Ciencias Empresariales \\ Universidad Católica de El Salvador, El Salvador
}

Fecha de recepción: $17-12-2019$

Fecha de aceptación: $20-02-2020$

\section{Resumen}

A nivel mundial, el sector turístico aumenta año con año el volumen de personas que viajan y el monto que invierten; El Salvador no es la excepción. El país ha potenciado sus esfuerzos con políticas, programas e inversión para apoyar al desarrollo del sector.

Una estrategia para las empresas es innovar para mejorar su competitividad en el mercado, por ello se realizó una investigación descriptiva que evaluó la temática en cuatro áreas: productos y servicios; procesos, organización y marketing.

El objetivo de la investigación fue estudiar los esfuerzos que realizan las empresas en el tema de innovación. Para ello se consultó a empresarios del sector a fin de elaborar un diagnóstico de las actividades relacionadas a la temática y sus fuentes de conocimiento. Se identificaron dos principales barreras para innovar: falta de recursos financieros y el alto costo de inversión.

Palabras clave: Innovación turística, procesos, bienes y productos, marketing, organización.

\begin{abstract}
Globally, the tourism sector increases year by year the volume of people who travel and the amount they invest; El Salvador is not the exception. The country has strengthened its efforts with policies, programs and investment to support the development of the sector.

A strategy for companies is to innovate to improve their competitiveness in the market. For this reason, a descriptive research was carried out that evaluated the subject in four areas: products and services; processes, organization and marketing.

The objective of the research was to study the efforts made by companies in the field of innovation. To do this, businesspersons from the sector were consulted in order to prepare a diagnosis of the activities related to the subject and their sources of knowledge. Two main barriers to innovation were identified: lack of financial resources and the high cost of investment.
\end{abstract}

Key words: Touristic innovation, processes, goods and products, marketing, organization.

1. Maestra en Asesoría Educativa, Docente investigadora; email: rocio.portillo1@catolica.edu.sv; ORCID: https://orcid. org/0000-0003-3505-5709

2. Maestro en Asesoría Educativa, Docente investigador; email: victor.quintana@catolica.edu.sv; ORCID: https://orcid. org/0000-0002-2597-6291

3. Maestra en Asesoría Educativa, Docente investigadora; email: ada.torres@catolica.edu.sv; ORCID: https://orcid.org/0000003-3261-1028 


\section{Introducción}

De acuerdo a la Organización Mundial del Turismo (2018), el turismo se ha convertido en un sector que se puede explotar en todos los sentidos, ya que es la tercera categoría de exportación más grande del mundo, debido a las variadas actividades que se pueden desempeñar, y los productos o servicios que se pueden integrar. Pero en muchos casos esto solamente se queda en un producto; no se modifica o no se desarrolla su potencial como, por ejemplo, diversificar los productos autóctonos de una región. Si se analizan las potencias mundiales, se puede ver la constante búsqueda de elementos que ayudan a mantener su cuota de mercado en el turismo, y su inversión para atraer más consumidores mediante nuevas modalidades.

Según la Escuela Superior de Administración y Dirección de Empresas (2017), a través de su Informe Estado de la Innovación Turística en España, la apertura de procesos de co-creación con clientes, el establecimiento de vínculos a través de las redes sociales y la inversión en estructura son las principales tendencias de desarrollo de la innovación en las empresas turísticas españolas. El mismo informe define que, en un $70 \%$ de los casos, los principales facilitadores para emprender son: el mentoring, la formación y la asistencia a seminarios y congresos. A estos le siguen la capacidad de liderazgo y trabajo en equipo (59\%); así como la comunicación $(42 \%)$.
Un ejemplo respecto al uso de la tecnología en el sector turístico lo muestra Argentina. Según Putruele (2017), este país cuenta con aplicaciones para orientar a los turistas en este rubro. Tal es el caso de la app BA Tours, que es una plataforma para que los usuarios puedan transaccionar bienes y servicios. Otro ejemplo es BA Turismo, que es un mapa interactivo que se puede usar sin wifi para que las personas puedan circular y tener toda la información agregada. Los ejecutivos del ente turístico en este país, además, lanzaron un observatorio que tiene relación con el índice de potencialidad turística; el cual mediría estratégicamente dónde se tienen que asignar recursos para buscar mercados. Este observatorio se encuentra en alianza con Segittur de España, el cual dispone de un programa de Big data ${ }^{4}$ para comprender el comportamiento de los consumidores de una manera más sofisticada (Freire, s.f.).

\section{Innovación en el sector turístico}

De acuerdo con Entorno Turístico (s.f.), “innovación es incluir nuevas ideas, productos, servicios y prácticas con la intención de ser más productivos, aprovechando al máximo los recursos disponibles: tiempo, materiales, espacios, etc.”. Por su parte, para Brandmanic (2018), la innovación turística no solo se refiere a innovación digital, sino también a la transformación cultural de las empresas turísticas, como pueden ser la capacidad de ser ágiles y afrontar el cambio para integrarlas en el modelo de gestión de cada día. 
Las redes sociales son de vital importancia para el sector turístico, pero se deben seleccionar cuáles y para qué tipo de clientes se tienen que utilizar. Nuevas tecnologías como la inteligencia aumentada, las tecnologías emocionales, machine, deep learning ${ }^{5}$, blockchain $^{6} \mathrm{y}$ movilidad autónoma son temas cada vez más presentes en las conversaciones de los responsables de innovación de las empresas turísticas (BrandManic, 2018).

Según Pons (2018), los elementos que debería contener una hoja de ruta de innovación turística incluyen

(...) tener una visión de 360 grados que englobe varios aspectos que afectan a toda la empresa, y que tienen que empezar en la dirección general de la misma; pero, aunque cada empresa debe ver qué aspectos son prioritarios, se podría hablar de los siguientes aspectos a trabajar:

- Gestión del cambio, ser flexibles y ágiles a los cambios.

- Apostar por la innovación abierta y la colaboración entre empresas.

- Fomentar alta inteligencia emocional y el talento creativo.

- Repensar el modelo de negocio, nuevas formas de financiación.

- Pasar del logo al branding, que ayude a posicionar la marca.
- Trabajar la creatividad con Speed Thinking, Design Thinking.

- Innovar en el marketing, la comunicación y el fomento del contenido.

- Intentar personalizarle el producto y la experiencia turística al cliente.

Para López de Ávila (2017),

la Inteligencia Turística será un hecho desde el momento en que comencemos a analizar los datos. La combinación de un mayor conocimiento del turista y las tecnologías móviles (Go mobile!), nos darán la oportunidad de llevar a cabo estrategias de marketing y promoción digital totalmente nuevas, disruptivas; enlazando con las emociones y necesidades de nuestros clientes y potenciales clientes de manera directa.

\section{Turismo en El Salvador}

En la Política Nacional de Turismo y Desarrollo Local de El Salvador (s.f.) se habla de tres ejes estratégicos, en donde el primero es la innovación creativa para el desarrollo local. Esta estrategia se orienta a convertir a los actores locales en protagonistas del desarrollo de sus comunidades, aprovechando sus iniciativas para incorporarlas a la planificación y articulación de la oferta turística en los destinos. De esta forma se optimizan las inversiones públicas y privadas, a través de un proceso de intercambio de conocimientos y asesoría técnica; así como también, identificar acciones que posibilitan un

5. Aprendizaje profundo. Algoritmos de aprendizaje profundo relacionados con la inteligencia artificial.

6. Registro de operaciones de organizaciones y empresas en tiempo real. 
mayor impacto del turismo en el corto, mediano y largo plazo.

De acuerdo al Ministerio de Turismo de El Salvador (2017):

Los indicadores de gestión de turismo en El Salvador muestran el crecimiento en el nivel de ingreso, siendo al cierre del año 2018 (US\$ \$1,532.61 millones de dólares) el más alto en los últimos catorce años. Igual perspectiva se tuvo en cuanto a la llegada de visitantes $(2,535,661$ miles $)$ con un crecimiento del $12.9 \%$, en relación a los datos del año 2017.

Esta misma institución refleja para el año 2018 que, entre los visitantes de ese año, el $66.1 \%$ fueron turistas y el 33.9\% excursionistas. De acuerdo a estos datos, el porcentaje de turistas que ingresan a El Salvador lo hacen vía terrestre $(57 \%)$ y vía aérea $(43 \%)$; mientras que los visitantes de día (excursionistas) entran vía terrestre $(99.7 \%)$ y marítima $(0.3 \%)$. En cuanto al aspecto de procedencia, estos son turistas provenientes de Centroamérica (57.3\%), Norteamérica $(36.2 \%)$, Suramérica $(3 \%)$, Europa $(2.5 \%)$, el Caribe $(0.3 \%)$ y otros países $(1 \%)$ (MITUR, 2018).

En El Salvador se tiende a visualizar segmentos de mercados en los cuales aún no se ha llegado a innovar, tales como: turismo para personas con capacidades especiales, personas con problemas motrices, turismo pet friendly y turismo contemplativo. Pero en los últimos diez años, se ha potenciado mucho a estos sectores; prueba de ello es la creación del Ministerio de Turismo (MITUR) como ente de gobierno que gestiona la política del sector.

El crecimiento del rubro turismo ha sido sostenido, pero no todo depende del gobierno, muchos de los esfuerzos deben ser del sector privado; por lo que es esencial hacer un diagnóstico para conocer la innovación que potencie a las empresas del sector. Ante esto es necesario responder la pregunta: ¿cuáles son los esfuerzos de innovación de las empresas del sector turismo en el país?

\section{Metodología}

La investigación de tipo descriptivo evaluó los esfuerzos de innovación adoptados por 42 empresas del sector turismo. Para ello se abarcaron diversos departamentos, tanto de la zona occidental (Santa Ana, Ahuachapán, Sonsonate) como departamentos de la zona central (La Libertad, San Salvador, Cuscatlán, La Paz y Cabañas).

Inicialmente, la población de estudio fueron 70 empresas, a quienes se les envió una encuesta online, constituida por preguntas abiertas, cerradas y de opción múltiple. De estas, solo se obtuvo el apoyo de 25 empresas del área de restaurantes, hoteles, tour operadores, servicios de catering, transporte turístico, agentes de viajes y organizadores de eventos. Aunado a esta cantidad inicial, para recolectar mayor información, se visitó a 17 empresas más para contestar el mismo cuestionario de forma presencial. La interacción realizada con los gerentes o 
encargados de las empresas ayudó a identificar insumos sobre la temática.

\section{Resultados}

La innovación de productos turísticos (bienes y servicios) se realiza basado en el seguimiento de otras empresas; es decir, por ideas fortuitas que resultan de observar a la competencia, o que tienen origen en las opiniones de los clientes; y no porque sean el resultado de laboratorios de innovación, de instituciones de educación superior, o de entes gubernamentales que apoyan el sector.

Las empresas hacen innovación más por el hecho de seguir compitiendo o de sobrevivir. La creación de nuevos productos o significativamente mejorados representa el 50\% del quehacer de las empresas. Por su parte, la otra mitad de los empresarios manifestó no realizar esta práctica. En cuanto al hecho de ofrecer servicios nuevos o mejorados, un 76\% de los empresarios contestó afirmativamente; es decir, el porcentaje restante $(24 \%)$ no lo realiza.

Respecto a la innovación dentro de los métodos de logística, entrega o distribución para sus insumos, bienes y servicios, el 54\% de los empresarios manifiesta que es necesario salir del viejo modelo de esperar que el cliente llegue al negocio, y proporcionar sus productos hasta la puerta de su casa. No obstante, un $46 \%$ piensa diferente.

También se midió la percepción de los empresarios turísticos en cuanto a las innovaciones de los procesos dentro de la empresa. Entre esto se resalta el compromiso de mejorar la calidad, de aumentar la gama de bienes y servicios, la flexibilidad y la necesidad de buscar nuevos mercados (ver figura 1).

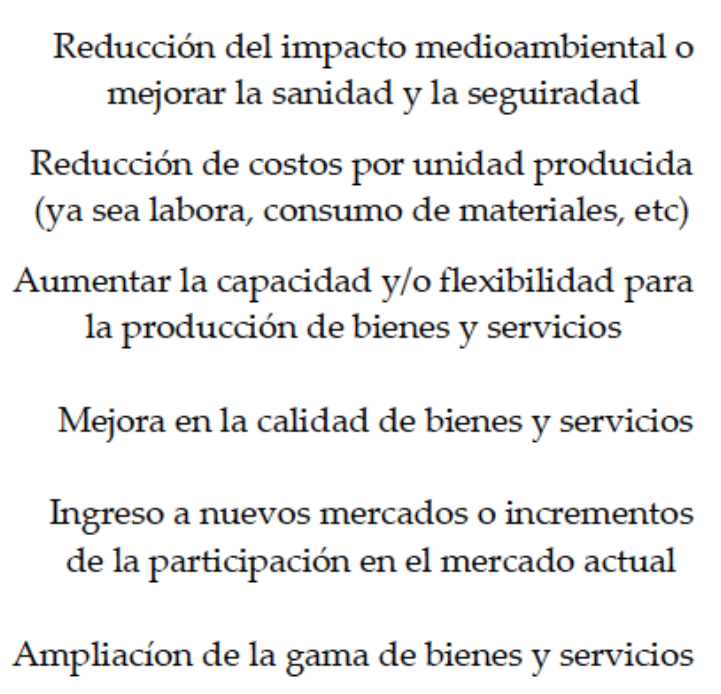

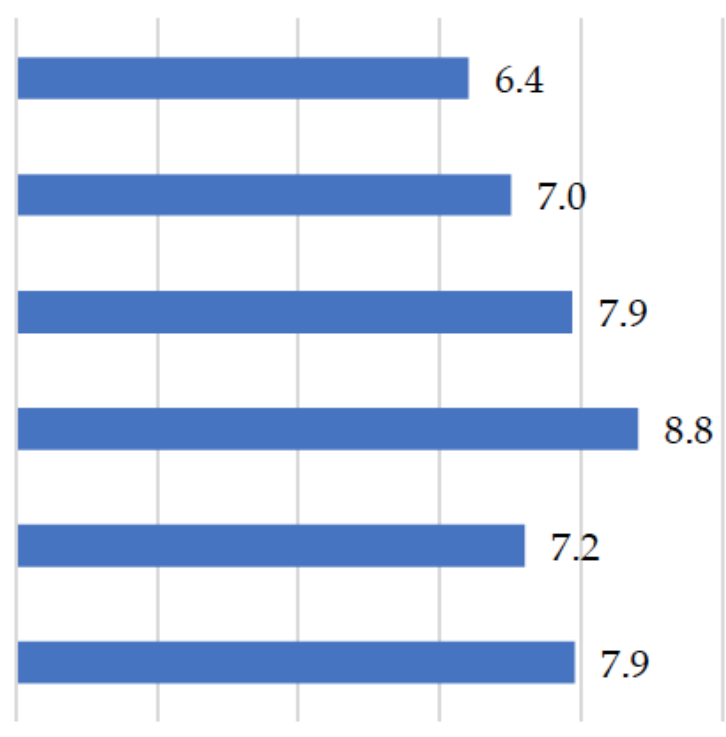

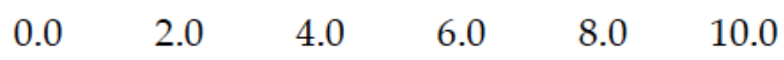

Figura 1. Percepción de los empresarios respecto a los efectos de las innovaciones de productos y servicios en los últimos dos años. 
Asimismo, se evaluaron las nuevas prácticas de negocios, encontrando que un $73 \%$ de las empresas han realizado prácticas innovadoras; y son conscientes que una mejor organización en los procesos busca mejorar la calidad de lo que ofrecen. Esto lo concluyeron a través de haber evaluado empíricamente el crecimiento de su competencia.

En cuanto a los métodos de organización y toma de decisiones, el $71 \%$ de las empresas turísticas han realizado prácticas innovadoras para mejorar los sistemas de gestión, reestructurarse y capacitar a sus empleados continuamente; contrario al 29\% de los consultados que no lo han realizado.

Respecto a los métodos de organización de las relaciones externas con otras empresas o instituciones públicas, el $68 \%$ de las empresas realizan este tipo de innovaciones en el área de turismo, a diferencia del porcentaje restante $(32 \%)$. Cabe señalar que el número de empleados que trabajan en las empresas están sujetos a los eventos que estas sirven. Por ello es primordial que las mismas estén aliadas en la subcontratación de personal.

La innovación implica mejorar la calidad, la cual va de la mano con el tiempo de respuesta para el cliente; así como mejorar la comunicación de todos aquellos que integran las empresas. La importancia de estos tres elementos es evidente en la figura 2.

La innovación de marketing se midió en el diseño, envase y embalaje de productos, en donde un $63 \%$ de los empresarios turísticos manifestaron haber realizado innovaciones; mientras que el 37\% negó la existencia de cambios significativos en estos aspectos.

\section{Mejorar la comunicación y/o participación de información dentro de sus empresa y/ocon otras empresas y/o instituciones}

Reducir los costos por unidad producidad

Mejorar la calidad de sus bienes y servicios

Mejorar la habilidad para desarrollar nuevos productos y o procesos

Reducir tiempo de respuesta a la necesidad del cliente o proveedor
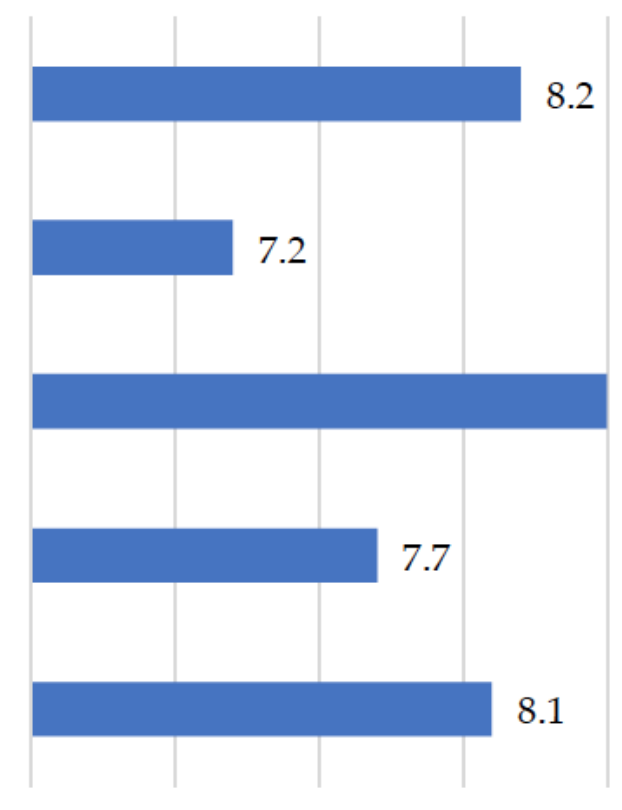

8.5

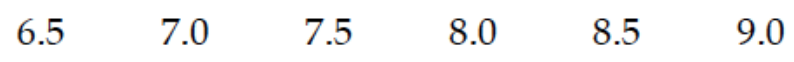

Figura 2. Efectos de la innovación organizacional realizada en los dos últimos años. 
Donde más se observaron oportunidades de innovación fue en la promoción de los productos, específicamente a través de internet, redes sociales, aplicaciones móviles, pago electrónico, etc. Ante esto, solo el $71 \%$ de las empresas realizan iniciativas para adoptar estas prácticas, mientras que el 19\% mantiene los medios y técnicas tradicionales (ver figura 3 ).

En cuanto a los efectos de la innovación, la nota más alta corresponde a incrementar la participación en el sector (8.0); o por lo menos, mantenerla. De ahí que la introducción de nuevos productos y servicios dentro de su oferta y buscar nuevos mercados para los mismos ocupa el segundo lugar; seguido de la introducción de productos en un nuevo mercado geográfico.

El internet es la fuente que más potencia las actividades de innovación en el sector turístico, seguido de las relaciones con los clientes, quienes demandan necesidades de productos $y$ servicios. Esto obliga a las empresas a brindar información de primera mano sobre su rubro (ver figura 4).

La falta de recursos financieros y el difícil acceso a créditos se vuelven un obstáculo importante para las empresas del sector. Este es un factor común en la micro y pequeña empresa a nivel general. Sin embargo, otro elemento a evaluar es el costo elevado de la innovación (ver figura 5).

En cuanto al factor del conocimiento, la falta de personal especializado o técnicos del sector, es el aspecto más sobresaliente (6.6). Es necesario formar personal tanto en la gestión, como operativos especializados en el rubro. En este aspecto juegan un papel relevante las instituciones de educación del país, ya que la ausencia de asociación y cooperación da como resultado una pobre gestión de la innovación, y el surgimiento de esfuerzos individuales por parte de las empresas (ver figura 6).

\section{Introducir productos para un mercado geográficamente nuevo}

Introducir productos para un nuevo segmento de mercado

Incrementar o mantener la participación del mercado

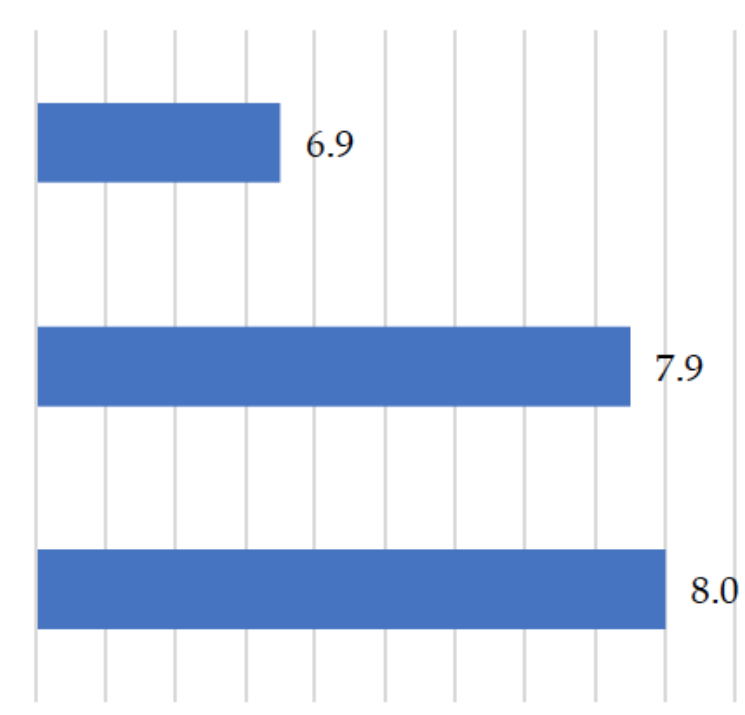

$\begin{array}{lllllllllll}6.2 & 6.4 & 6.6 & 6.8 & 7.0 & 7.2 & 7.4 & 7.6 & 7.8 & 8.0 & 8.2\end{array}$

Figura 3. Efectos de la innovación de marketing realizada en los dos últimos años. 


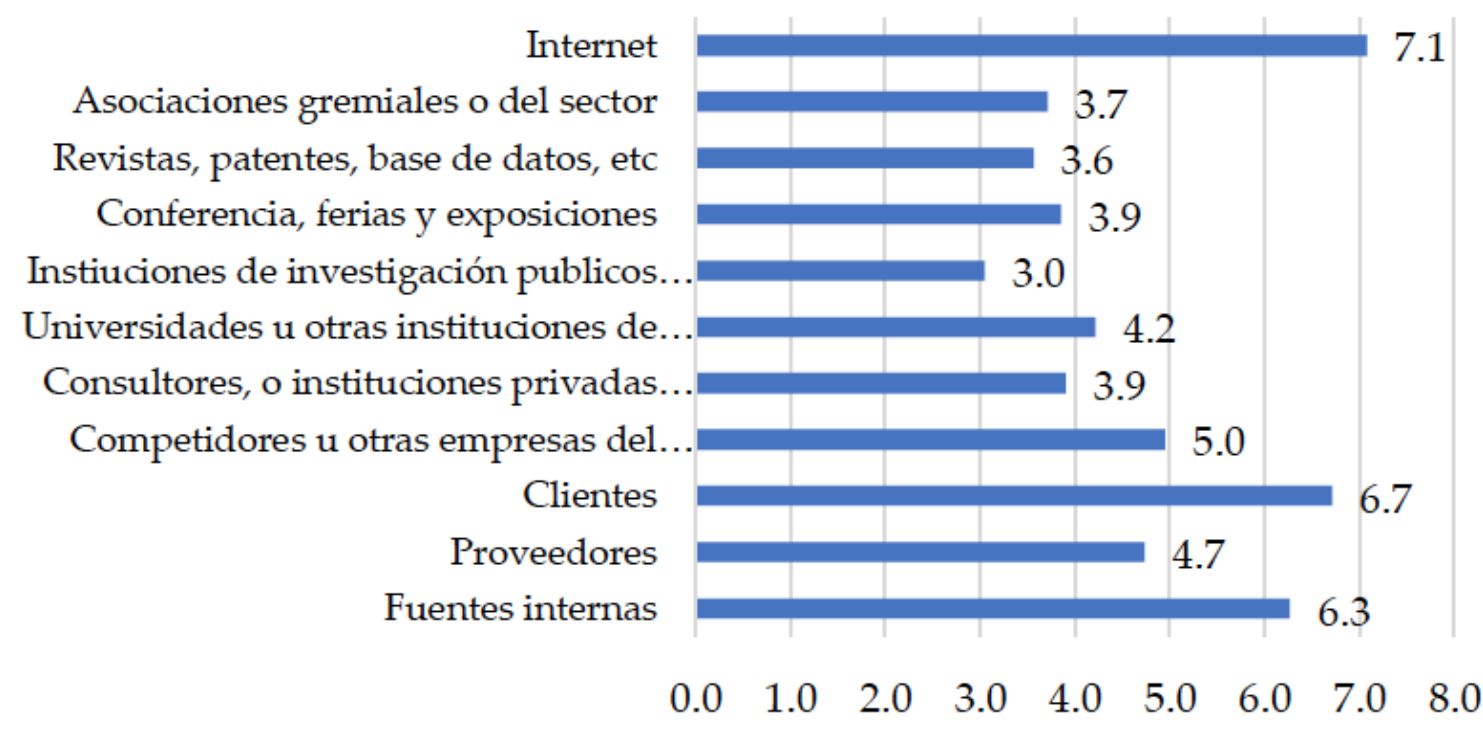

Figura 4. Fuentes de información en actividades innovadoras.

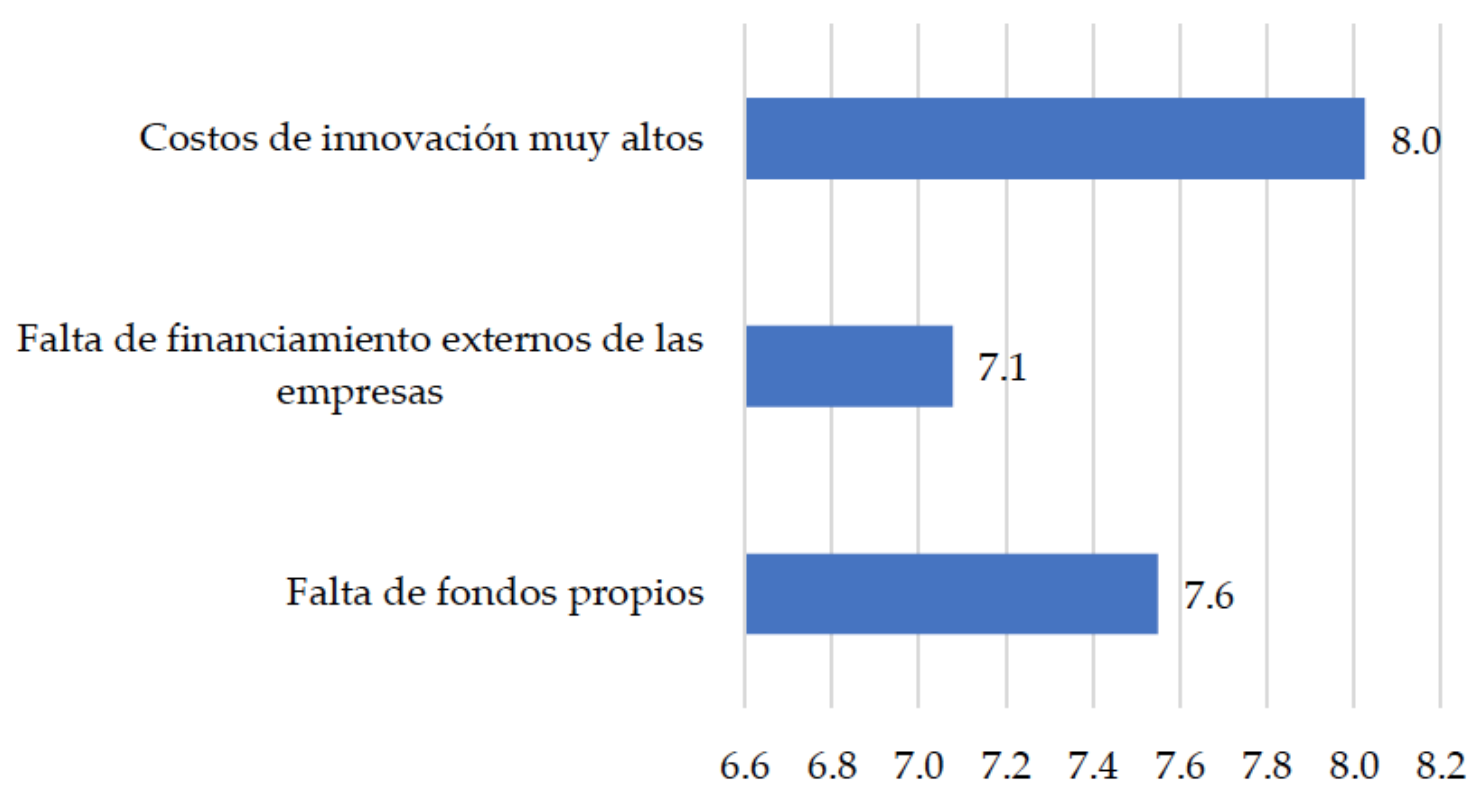

Figura 5. Factores de costo de la innovación.

Las empresas no definen el mercado en cuanto Los empresarios perciben que la demanda de a la demanda de los mismos, ya que se adap- productos innovados no afecta al negocio, detan a lo que los clientes les exigen, respecto a bido a que las personas demandan los servicios productos o servicios turísticos; por lo que las según la oferta ya establecida; y muchos exgrandes empresas tienen más probabilidad de tranjeros arriban al país con los servicios concaptar turistas por tener una mejor consolida- tratados (ver figura 8). ción en la gestión del mercado ( ver figura 7). 
Dificultad en encontrar cooperación de parnets para la innovación

Falta de información de los mercados

Falta de información sobre la tecnología

Falta de personal calificado

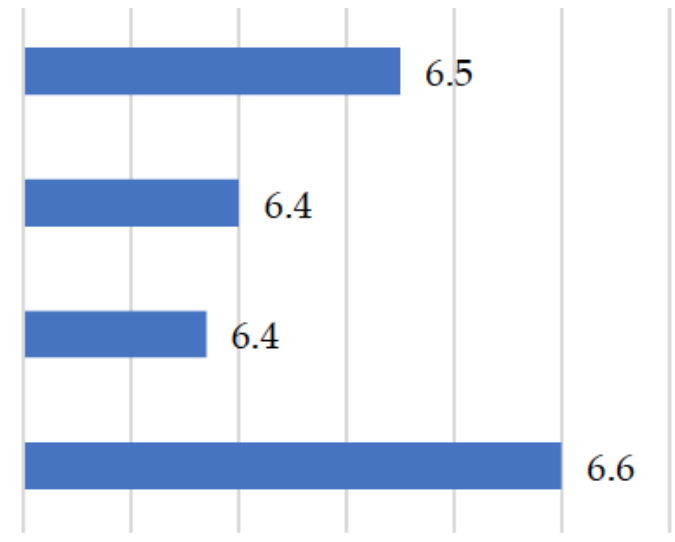
6.3
6.4
6.4
6.5
$6.5 \quad 6.6$
6.6

Figura 6. Factores vinculados al conocimiento.

Incertidumbre respecto a la demanda de bienes o servicios innovados

Mercado dominado por empresas establecidad
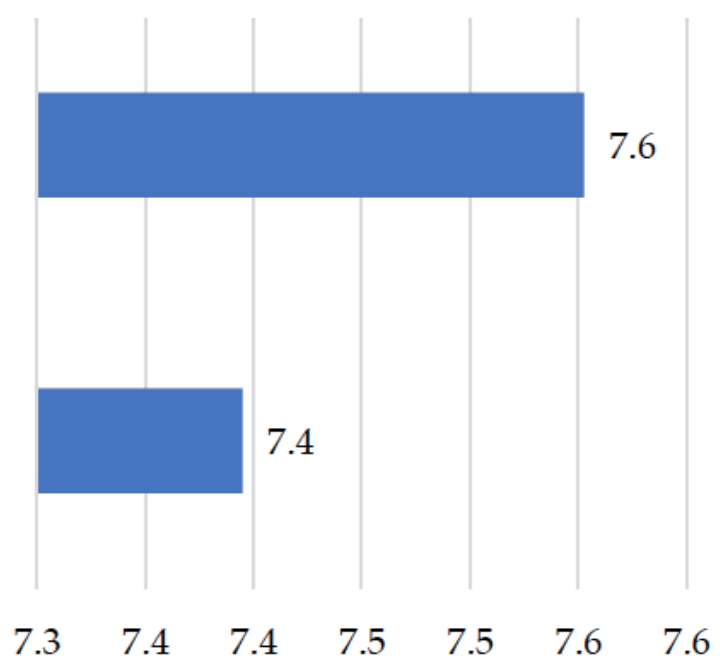

Figura 7. Factores de mercado turístico.

\section{Dificultad regulatoria \\ No es necesario por falta de demanda de innovaciones \\ No es necesario debido a innovaciones previas}
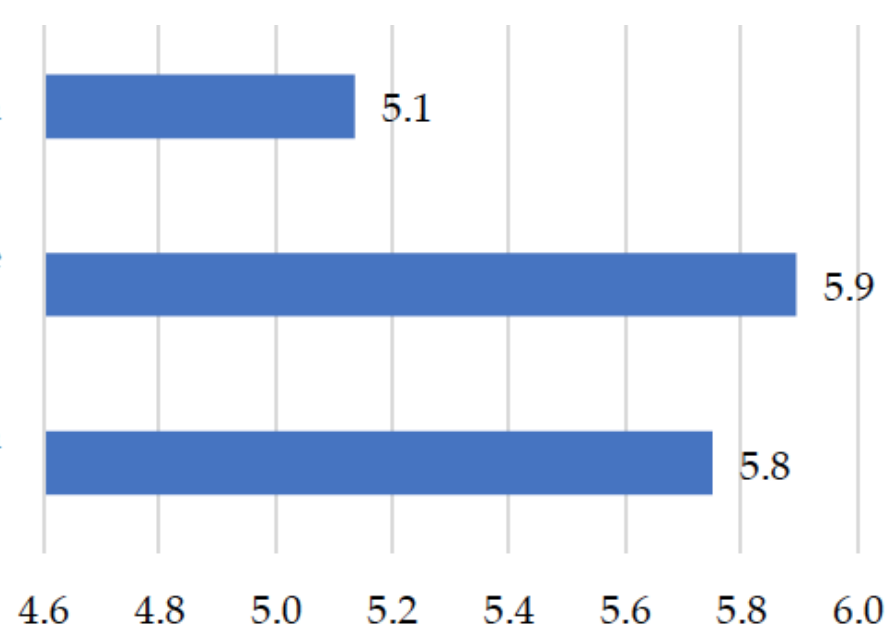

Figura 8. Factores de demanda de servicios o productos turísticos. 


\section{Discusión}

Las empresas que forman parte del sector turismo reconocen que la innovación es una estrategia de competitividad, pero también de sobrevivencia dentro del mercado. Dos elementos dentro de la innovación son los productos y el mercadeo; en los cuales se puede basar una estrategia para mejorar la oferta a las personas que están interesadas en visitar el país. Un elemento que se puede tomar en cuenta, al momento de desarrollar productos y servicios, es la extensión del territorio salvadoreño, ya que el tamaño del país posibilita el hecho de visitar diversos destinos el mismo día.

La investigación evidenció que las fuentes de innovación se generan desde el interior de la empresa; pero también es necesario el aporte de las instituciones de educación superior en cuanto a la articulación academia-sector empresarial para promover el desarrollo de la innovación. Esto refleja la necesidad de un punto de encuentro entre ambos sectores para la formación de los trabajadores especializados, quienes potencien la innovación. Así como también, el fomento de emprendimientos dentro del sector turístico, que tengan como base la innovación, la tecnología y el crecimiento.
Otro elemento limitado es la escasa oportunidad de asociación para propiciar trabajo colaborativo, lo cual genera esfuerzos individuales por parte de las empresas; dando como resultado, una pobre gestión de la innovación. A pesar de eso, existen fuentes de innovación que pueden aprovecharse, tales como el conocimiento de la oferta de otras empresas, la asistencia a ferias de emprendimiento o la investigación a través de internet, redes sociales, entre otros. De esta forma se evitaría la ausencia de información respecto a los perfiles de turistas, sus preferencias, áreas de consumo de productos y servicios, etc. Esto es importante, ya que se pueden clasificar las características y gustos no solo por país, sino también por generación (Babyboomers, Millennials, Generación Z, y subsiguientes).

En conclusión, para combatir los obstáculos que evitan la generación de innovación (liquidez financiera, el difícil acceso a créditos y la falta de personal calificado), los gestores empresariales deben estar atentos a las nuevas tendencias y adoptarlas dentro de su cometido; pero también deben mejorar las relaciones con los distintos sectores involucrados, ya que entre más colaboración exista, mejores productos o servicios se ofrecerán. 


\section{Referencias}

Álvarez, A.; Rego, G.; Leira, J.; Gomis, A.; Caramés, R. y Andrades, M. J. (2008). Innovación turística: perspectivas teóricas y objetos de estudio. Rotur/Revista de Ocio y Turismo, (1), 1950. Recuperado de https://www.researchgate.net/publication/228462715_Innovacion_turistica_perspectivas_teoricas_y_objetos_de_estudio

Aula Internacional de Innovación Turística ESADE Business School (2017). Informe innovación turística en España 2017. Recuperado de https://www.ithotelero.com/wp-content/ uploads/2017/07/Informe-AIIT-2017.pdf

BrandManic (20 de enero de 2018). Fitur 2018: Claves de la innovación turística [Mensaje en un blog]. Recuperado de https://www.brandmanic.com/innovacion-turistica/

Comunidad de Madrid (s.f.). Estrategia de Turismo 2016-2019. Recuperado de http://www.madrid.org/cs/Satellite?blobcol=urldata\&blobheader=application/pdf\&blobheadername1=Content-Disposition\&blobheadervalue $1=$ filename\%3DEstrategiaTurismoCAM_vDEF.PDF\&blobkey $=\mathrm{id} \&$ blobtable $=$ MungoBlobs\&blobwhere $=1352913096654 \&$ ssbinary $=$ true

ESADE Bussines School (junio, 2017). Informe Innovación Turística en España 2017. Recuperado de https:/www.ithotelero.com/wp-content/uploads/2017/07/Informe-AIIT-2017.pdf

Fundación Orange (2016). La transformación digital en el sector turístico. Recuperado de: http:// www.fundacionorange.es/wp-content/uploads/2016/05/eE_La_transformacion_digital_del_ sector_turistico.pdf

Gobierno de Chile (s.f.). Estrategia nacional de turismo 2012-2020. Recuperado de https://ccps. mma.gob.cl/wp-content/uploads/2016/01/1_Estrategia-Nacional-de-Turismo-2012-2020.pdf

Junta de Andalucía, Dirección General de Innovación y Prospectiva Turística (s.f.). Estrategias para la innovación tecnológica del sector turístico andaluz. Recuperado de http://www.pymesonline.com/uploads/tx_icticontent/R02553_turismo.pdf

Ministerio de Turismo de El Salvador (s.f.). Turismo y Desarrollo Local: Política Nacional de Turismo. Recuperado de www.transparencia.gob.sv

Ministerio de Turismo de El Salvador (2017). Política de turismo sostenible. Recuperado de http://www.mitur.gob.sv/wp-content/uploads/2017/12/POLITICA-DE-TURISMO-SOSTENIBLE-DE-LA-REPUBLICA-DE-EL-SALVADOR-20171.pdf

Ministerio de Turismo de El Salvador (2018). Informe Estadístico Enero-diciembre 2018 Preliminar. Recuperado de www.transparencia.gob.sv 
Oficina Estadística de las Comunidades Europeas y Organización para la Cooperación y el Desarrollo Económico (2006). Manual de Oslo: Guía para la recogida e interpretación de datos sobre innovación. Recuperado de http://www.itq.edu.mx/convocatorias/manualdeoslo.pdf

Organización Mundial del Turismo (2018). Notas metodológicas de la base de datos de estadísticas de turismo. Recuperado de https:/www.unwto.org/es/news/2018-04-04/notas-metodologicas-de-la-base-de-datos-de-estadisticas-de-turismo-edicion-2018

Organización Mundial del Turismo (2019a). Global and Regional Tourism Performance. Recuperado de https://www.unwto.org/global-and-regional-tourism-performance

Organización Mundial del Turismo (2019b). Tourism's Growth across all regions strengthens sector's potential to contribute to sustainable development agenda. Recuperado de https://www. unwto.org/news/tourisms-growth-across-all-regions-strengthens-sectors-potential-to-contribute-to-sustainable-development-agenda

Redacción (s.f.). Negocios Turísticos: las oportunidades que tenemos los mexicanos. Entorno Turístico: Hablemos de Turismo. Recuperado de https://www.entornoturistico.com/negocios-turisticos-las-oportunidades-que-tenemos-los-mexicanos/

Redacción (2019, 18 de enero). Los países que más invierten en investigación e innovación. $D i$ nero. Recuperado de https://www.dinero.com/edicionimpresa/mundo/articulo/paises-que-invierten-en-innovacion/254269

Restrepo, J.; Franky, J.; Vásquez, M. y Fajardo, K. (2018). Plan Nacional Sectorial de turismo 2018 - 2022. Recuperado de https:/www.academia.edu/37607682/Plan_Nacional_Sectorial_ del_Turismo_2018_-2022

Socateli, M. (s.f.). Desarrollo de productos turísticos innovadores. Recuperado de http://www. ucipfg.com/Repositorio/MGTS/MGTS14/MGTSV-09/semana2/Mercadeo_Turistico_-_ LS2.3._Desarrollo_de_Productos_Turisticos.pdf

Subsecretaría de planeación y política turística de México (2017). Estrategia para el Impulso y Desarrollo del Turismo de Naturaleza. Recuperado de https://www.gob.mx/cms/uploads/ attachment/file/278215/ETNM_v3_oct17.pdf

Taboada, J. (s.f.). La semilla de la innovación en el turismo tradicional de El Salvador. TYS Magazine. Recuperado de https://www.tysmagazine.com/la-semilla-de-la-innovacion-en-el-turismo-tradicional-de-el-salvador-centroamerica/

Teos, E. (11 de marzo de 2019). El turismo en El Salvador creció 12.9\% en 2018. La prensa gráfica. Recuperado de https://www.laprensagrafica.com/economia/El-turismo-en-El-Salvadorcrecio-12.9--en-2018-20190110-0373.html 